\title{
Anti-hypertensive effect of crocin and hesperidin combination in high-fat diet treated rats
}

\author{
MAHMOUD HASHEMZAEI ${ }^{1}$, RAMIN REZAEE ${ }^{2,3}$, MOJTABA NABATZEHI ${ }^{1}$, \\ KONSTANTINOS TSAROUHAS ${ }^{4}$, TAXIARCHIS KONSTANTINOS NIKOLOUZAKIS ${ }^{5}$, \\ GEORGE LAZOPOULOS $^{6}$, DEMETRIOS A. SPANDIDOS ${ }^{7}$, ARISTIDIS TSATSAKIS ${ }^{5}$ and JAFAR SHAHRAKI ${ }^{1}$ \\ ${ }^{1}$ Department of Pharmacology and Toxicology, Faculty of Pharmacy, Zabol University of Medical Sciences, \\ 9861615881 Zabol; ${ }^{2}$ Clinical Research Unit, Faculty of Medicine; ${ }^{3}$ Neurogenic Inflammation Research Center, \\ Mashhad University of Medical Sciences, 9177948564 Mashhad, Iran; ${ }^{4}$ Department of Cardiology, \\ University Hospital of Larissa, 41110 Larissa; ${ }^{5}$ Center of Toxicology Science and Research, Medical School, \\ University of Crete; ${ }^{6}$ Department of Cardiothoracic Surgery, University General Hospital of Heraklion, Medical School, \\ University of Crete; ${ }^{7}$ Laboratory of Clinical Virology, School of Medicine, University of Crete, 71003 Heraklion, Greece
}

Received March 2,2020; Accepted April 7, 2020

DOI: $10.3892 /$ etm.2020.8650

\begin{abstract}
Cardiovascular diseases are the leading cause of death worldwide. Hypertension is the most important cause of such conditions. The use of medicinal herbs is of particular importance due to their lower cost and side effects. The aim of the present study was to compare the effect of hesperidin (HES) and crocin (CRO) alone and in combination, on blood pressure in a rat model of high-fat diet (HFD)-induced hypertension, using invasive carotid artery measurements. Animals were randomly assigned to the following groups: control group (received standard chow diet), HFD control group (received HFD containing $32 \%$ kcal of fat and $0.1 \%$ cholesterol), and three groups of HFD-treated animals that were treated with a single dose of CRO $(20 \mathrm{mg} / \mathrm{kg})$, HES $(20 \mathrm{mg} / \mathrm{kg})$, or CRO + HES $(20+20 \mathrm{mg} / \mathrm{kg})$. Except for the control group, rats received HFD for 7 weeks. On day 50, CRO, HES and normal saline were administered intraperitoneally and carotid arteries of the rats were cannulated. Three hours after the carotid artery cannulation, mean arterial blood pressure (MAP), systolic and diastolic blood pressure (SBP and DBP), and heart rate (HR) were measured using an intra-arterial catheter with the use of a Power Lab
\end{abstract}

Correspondence to: Professor Aristidis Tsatsakis, Center of Toxicology Science and Research, Medical School, University of Crete, 71003 Heraklion, Greece

E-mail: tsatsaka@uoc.gr

Professor Jafar Shahraki, Department of Pharmacology and Toxicology, Faculty of Pharmacy, Zabol University of Medical Sciences, 9861615881 Zabol, Iran

E-mail: jafar.shahraki@gmail.com

Key words: crocin, hesperidin, blood pressure, rats, high-fat diet, hypertension system. Data was analyzed using SPSS software. Rats that received HFD for 49 days presented a significant increase in SBP, HR and MAP compared to the control group $(\mathrm{P}<0.001)$. Whereas, HFD-treated rats of the CRO + HES group showed lower levels of SBP, HR and MAP; however, DBP remained unaffected. HES administration in HFD treated rats resulted in a significant decrease in SBP compared to the HFD control group with no significant differences in MAP. The hypotensive effects of the simultaneous administration of CRO and HES in HFD-hypertensive rats suggest the need for further study of these two natural products as a potential preventive measure against hypertension development, especially in patients with high normal blood pressure.

\section{Introduction}

Natural products are small molecules produced by organisms as primary and secondary metabolites and they exert various useful properties including anti-oxidant, anti-inflammatory, cancer preventive, cardiovascular, anti-Alzheimer's, platelet modulatory and pain relief effects (1-5).

These compounds are considered new therapeutic tools for the management of cardiovascular diseases, and they have received considerable attention. In this context, increasing number of reports indicated beneficial effects of foods with high levels of polyphenols (fruits, tea and cocoa) against cardiovascular diseases (6-9) in humans and similar favorable effects were noted for isolated flavonoids in animal models $(10,11)$.

Crocin (CRO), a carotenoid from the tetraterpenes family, is responsible for the unique color of saffron, stigmas of Crocus sativus (12). Considerable amount of evidence has confirmed that CRO exhibits antioxidant, radical scavenging, cytotoxic and antitumor $(12,13)$, renoprotective (14) and anti-hyperlipidemic (15) effects. Several studies have shown the beneficial effects of this compound against cardiovascular conditions as it could alleviate blood biomarkers associated with obesity (16), had protective effects against angiotensin II-hypertension (17) 
and normalized the blood pressure of rats chronically administered with desoxycorticosterone acetate (18).

In a study, CRO reduced mean arterial blood pressure (MAP) in rats and its effects were more marked in hypertensive animals compared to normotensive ones (19). It was noted that CRO inhibitory effects on the extracellular $\mathrm{Ca}^{2+}$ influx along with $\mathrm{Ca}^{2+}$ cytosolic release from intracellular $\mathrm{Ca}^{2+}$ supplies in the endoplasmic reticulum, might underlie its hypotensive effects induced by relaxing blood vessels $(20,21)$.

Citrus fruits contain flavanone glycosides hesperidin (HES) and naringin which are members of the bigger family of flavonoids that comprises a large number of phenolic compounds. Flavanone glycosides were shown to have higher bioavailability compared to other flavonoid compounds. HES has shown various therapeutic properties such as anti-oxidant, anti-inflammatory, and anti-aging effects (22-26). Although the beneficial effects of HES on the cardiovascular system were shown by the significant reductions observed in the blood pressure and serum lipids following its administration (27-29), there is still debate regarding its value in this field since there are reports indicating that HES has no significant effect on the said cardiovascular parameters $(30,31)$.

It was reported that 25-week administration of HES reduced blood pressure and heart rate (HR) in spontaneously hypertensive rats but in normotensive Wistar-Kyoto rats, no such effect was observed (32). Moreover, a randomized, controlled, crossover study in healthy volunteers showed that supplementation with HES during a 4-week period, led to a significant reduction in diastolic blood pressure (DBP) (29).

In another study, glucosyl HES (G-HES), a water-soluble derivative of HES, and hesperetin, aglycone HES, were given to spontaneously hypertensive rats (SHR); both compounds reduced systolic blood pressure (SBP) which was significantly inhibited by a nitric oxide synthase inhibitor, N(G)-nitro-L-arginine methyl ester (33). Nevertheless, a recent meta-analysis of randomized controlled trials revealed that HES supplementation had no effect on SBP or DBP (28).

In the present study, effects of CRO and HES supplementation, alone and in combination, on MAP, SBP, DBP, and HR were assessed in rats treated with a high-fat diet (HFD) for 7 weeks.

\section{Materials and methods}

Animals. In this study, 40 male Wistar rats (aged 8-10 weeks; 200-250 g body weight, obtained from Animal Center of Zabol University of Medical Sciences, Zabol, Iran) were kept under standard conditions (at $25^{\circ} \mathrm{C}$ with $12 / 12 \mathrm{~h}$ light/dark cycles) and they had free access to food and water, ad libitum. All animal experiments were approved by the animal research Ethics Committee of Zabol University of Medical Sciences (Ethics committee approval no. IR. ZBMU.REC.1398.091) and performed in accordance with National Institute of Health Guide for the Care and Use of the Laboratory Animals.

Animals were randomly assigned to the following five groups (8 animals in each group): control group [rats received standard chow diet (CD; Harlan TD.7012) for 7 weeks + normal saline on day 50], HFD control group [received HFD containing $32 \% \mathrm{kcal}$ of fat and $0.1 \%$ cholesterol (Harlan TD.88137) for 7 weeks and normal saline on day 50 (34)], and three groups of HFD-treated animals that received a single dose of either CRO $(20 \mathrm{mg} / \mathrm{kg}), \mathrm{HES}(20 \mathrm{mg} / \mathrm{kg})$, or CRO + HES $(20+20 \mathrm{mg} / \mathrm{kg})$ on day 50 .

Materials. HES and CRO were purchased from Merck KGaA. Ketamine (Alfasan) and xylazine (Pantex Holland B.V.) were employed for induction of anesthesia. For measurement of SBP, DBP, HR and MAP, Power Lab (AD Instruments) was used $(35,36)$.

Animal diets. Animals were assigned into the two diet groups. The control group received purified low-fat diet (containing $10.6 \% \mathrm{kcal}$ as fat) for 7 weeks. The rest of the animals received HFD diet containing $32 \% \mathrm{kcal}$ as fat and $0.1 \%$ cholesterol, for 7 weeks (37). HFD was provided from Razi Institute, Tehran, Iran. The HFD animals were divided into HFD control group and CRO or HES or CRO + HES groups.

Methods. After the 7-week period of HFD, animals were fasted overnight (minimum period of 8-10 h), and anesthetized using ketamine/xylazine. The reflexes of the animals were checked, and they were placed on a suitable rodent surgical table with electrocardiogram (ECG) recording. The ventral side of the neck, right hind leg, and chest of the animals were carefully disinfected and shaved. A small incision $(1.5-2 \mathrm{~cm})$ was made in the neck of the rats for the tracheostomy procedure and carotid artery cannulation. Tracheostomy was performed using a small piece of pediatric Ryle's tube or rodent tracheal intubation tube (36). The carotid artery was identified and the cardiac end of the blood vessel was clamped by a bulldog clamp for cannulation (36). The blood vessel was cannulated employing a cannula pre-filled with heparinized normal saline $(0.5 \mathrm{IU} / \mathrm{ml})$. After cannulation, the bulldog clamp at the cardiac end of the blood vessel, was released slowly (9).

Three hours before cannulation, CRO and HES alone or in combination in the respective treatment groups or normal saline in the control and HFD-hypertensive group, were injected intraperitoneally (ip). After cannulation, the sensor was connected to the Power Lab instrument and the blood pressure, HR and ECG were recorded and analyzed using Lab Chart 8 software (36).

Statistical analysis. Data analysis was performed using SPSS version 11.5 (SPSS, Inc.). Differences among the groups were assessed using One-way ANOVA followed by Tukey's post hoc test. $\mathrm{P}<0.05$ was considered to indicate a statistically significant difference.

\section{Results}

Effects of HES and CRO on SBP in HFD-hypertensive rats. As presented in Fig. 1, HFD-treated animals showed significant increases in SBP compared to the control group $(\mathrm{P}<0.001)$. In the HES and HES+CRO groups, SBP was reduced significantly in comparison to the HFD control group (for both cases $\mathrm{P}<0.001)$. HES treatment resulted in a significant decrease of 


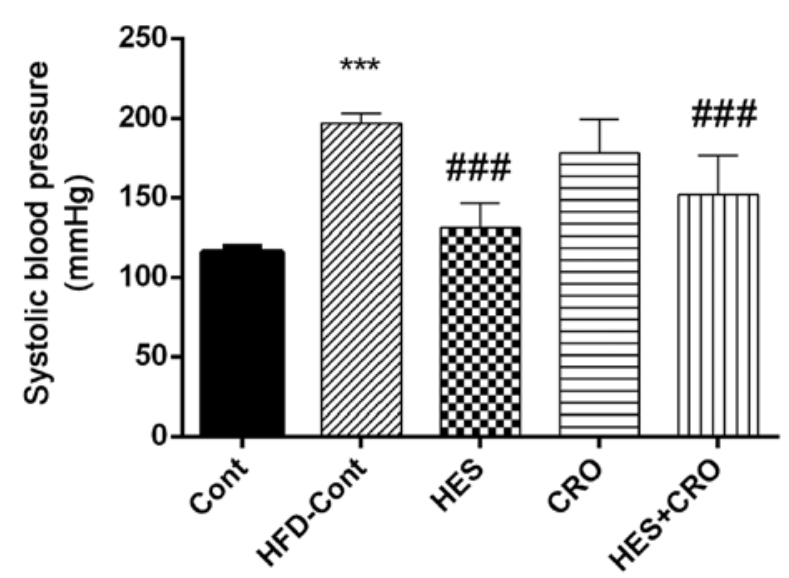

Figure 1. Effects of HES, CRO and HES+CRO on systolic blood pressure. ${ }^{* * * *} \mathrm{P}<0.001$ compared to the control group and ${ }^{\# \#} \mathrm{P}<0.001$ compared to the HFD-Cont group. Cont, control group; HFD-Cont, HFD-treated control rats that were treated with normal saline; HES, hesperidin; CRO, crocin.

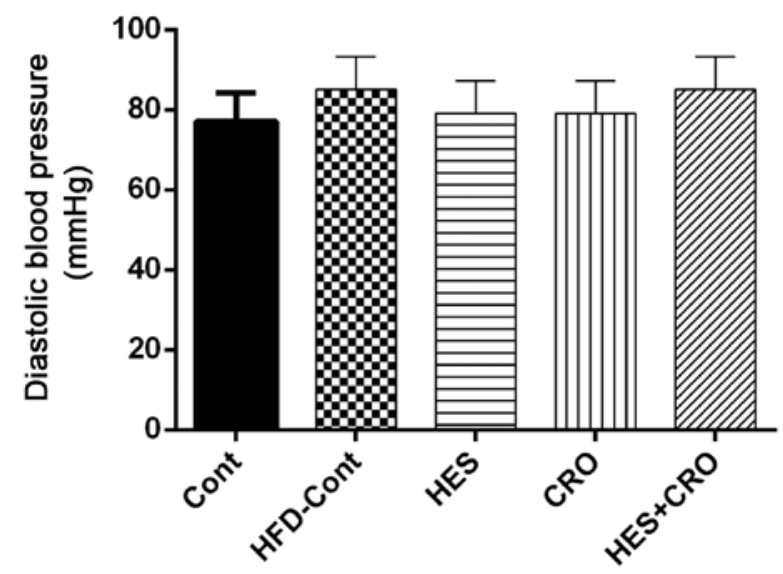

Figure 2. Effects of HES, CRO and HES+CRO on diastolic blood pressure compared to the HFD-treated control rats. Cont, control group; HFD-Cont, HFD-treated control rats that were treated with normal saline; HES, hesperidin; CRO, crocin.

SBP compared to the HFD control group. On the other hand, treatment of hypertensive animals with CRO alone did not reduce $\mathrm{SBP}$.

Effects of HES and CRO on DBP in HFD-hypertensive rats. As shown in Fig. 2, DBP did not show significant variations among the different groups.

Effects of HES and CRO on HR in HFD-hypertensive rats. Fig. 3 shows that HFD rats did not present a significant difference in HR compared to the control group which did not receive HFD. Nonetheless, HES+CRO treated animals showed a significant reduction in HR in comparison to the HFD control group $(\mathrm{P}<0.001)$.

Effects of HES and CROC on MAP in HFD-hypertensive rats. 7-week treatment of rats with HFD led to a significant increase in MAP compared to the control group $(\mathrm{P}<0.001)$. Furthermore, our results showed that HES+CRO administration in HFD rats significantly attenuated MAP compared to the HFD control group $(\mathrm{P}<0.001)$; however, treatment with

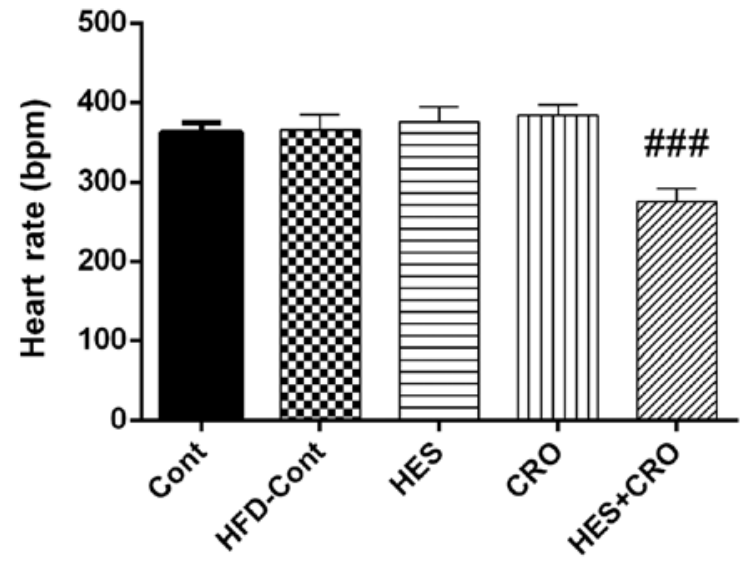

Figure 3. Effects of HES, CRO and HES+CRO on heart rate compared to the HFD-treated control rats. ${ }^{\# \#} \mathrm{P}<0.001$ in comparison to the HFD-Cont group. Cont, control group; HFD-Cont, HFD-treated control rats that were treated with normal saline; HES, hesperidin; CRO, crocin.

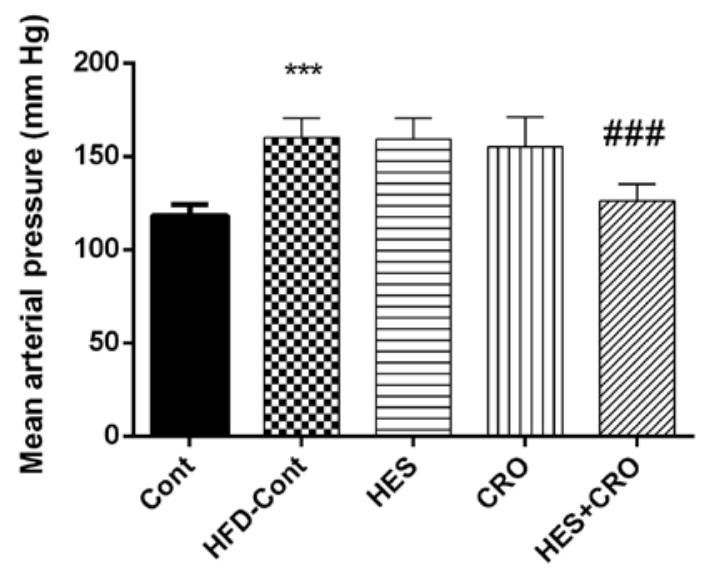

Figure 4. Effects of HES, CRO and HES+CRO on mean arterial blood pressure as compared to the control group, in HFD-hypertensive in rats. ${ }^{* * *} \mathrm{P}<0.001$ compared to the control group and ${ }^{\# \#} \mathrm{P}<0.001$ compared to HFD-treated control rats. Cont, control group; HFD-Cont, HFD-treated control rats that were treated with normal saline; HES, hesperidin; CRO, crocin.

HES or CRO alone did not result in significant alterations in MAP compared to the HFD control group (Fig. 4).

\section{Discussion}

In the current study, 7-week administration of HFD in rats induced systolic hypertension and increased MAP. Administration of a single dose of HES reduced high SBP. HES and the combination of HES and CRO significantly decreased SBP. HFD did not affect DBP or HR; however, it significantly increased MAP, which in turn, was significantly decreased by treatment with the combination of HES and CRO. To the best of our knowledge, this is the first experiment investigating the effect of co-administration of two natural products, HES and $\mathrm{CRO}$, in a rat model of HFD-induced hypertension and reports their effects on blood pressure and HR. Co-administration of these compounds seems to have synergistic effects.

It was reported that hypotensive effects of HES are induced through nitric oxide (NO)-mediated vasodilation (33). In a study by Yamamoto et al (33), it was shown that short-term 
administration of HES reduces SBP by NO-induced vascular relaxation. Several studies similarly confirmed the aforementioned mechanism of action of HES $(31,38,39)$. Another report confirmed that HES acutely stimulates phosphorylation of endothelial NO synthase to produce NO and cause vasodilation (31) and these results are in agreement with the data obtained in the present study on the short-term antihypertensive effects of HES (31). We found that HES alone reduced SBP, but HES in combination with CRO reduced simultaneously SBP, MAP and HR confirming the synergistic effects of HES and CRO in reduction of high blood pressure.

Previous studies showed that CRO can reduce hypertension in animal models $(18,40)$. Besides, there are other reports on the antihypertensive effects of other active ingredients and extracts of Crocus sativus (41-43). Some of these studies examined possible acute antihypertensive effects of CRO and crocetin $(40,42,43)$. In this context, Shafei et al $(40)$ showed that CRO 50, 100 and $200 \mathrm{mg} / \mathrm{kg}$ attenuated MAP, SBP and HR induced by angiotensin II. Our results showed that CRO $20 \mathrm{mg} / \mathrm{kg}$ did not alleviate hypertension induced by HFD. In HES + CRO $(20+20 \mathrm{mg} / \mathrm{kg})$-treated animals, a significant decrement of SBP and MAP was observed. In the HES $(20 \mathrm{mg} / \mathrm{kg})$-only treated group, though SBP was decreased, no changes in DBP, HR and MAP were found. Our results point to a potentially interesting synergistic effect for these natural products that might act via similar pathways (i.e., via NO-associated mechanisms) $(33,40)$.

In our study, the co-administration of HES and CRO exhibited favorable antihypertensive effects. The beneficial effects of these nutraceuticals could be further tested in clinical settings for the prevention and treatment of hypertension, especially in patients presenting with borderline stage 1 hypertension.

\section{Acknowledgements}

The present study was part of a Pharm. D. thesis and authors wish to thank Zabol University of Medical Sciences for support.

\section{Funding}

This work was funded by Zabol University of Medical Sciences and Students Research Committee of Zabol University of Medical Sciences.

\section{Availability of data and materials}

Not applicable.

\section{Authors' contributions}

MH and RR conceived and designed, and supervised the study. MN performed the experiments and collected the data and TKN analyzed the data. GL, KT, AT, JS and DAS interpreted the data and prepared the manuscript. All authors read and approved the final version of the manuscript.

\section{Ethics approval and consent to participate}

All animal experiments were approved by the animal research Ethics Committee of Zabol University of Medical Sciences (Ethics committee approval no. IR.ZBMU.REC.1398.091).

\section{Patient consent for publication}

Not applicable.

\section{Competing interests}

DAS is the Editor-in-Chief for the journal, but had no personal involvement in the reviewing process, or any influence in terms of adjudicating on the final decision, for this article. The other authors declare that they have no competing interests.

\section{References}

1. Hashemzaei M, Abdollahzadeh M, Iranshahi M, Golmakani E, Rezaee $\mathrm{R}$ and Tabrizian K: Effects of luteolin and luteolin-morphine co-administration on acute and chronic pain and sciatic nerve ligated-induced neuropathy in mice. J Complement Integr Med 14: 20160066, 2017.

2. Hashemzaei M, Imen Shahidi M, Moallem SA, Abnous K, Ghorbani $\mathrm{M}$ and Mohamadpour AH: Modulation of JAK2, STAT3 and Akt1 proteins by granulocyte colony stimulating factor following carbon monoxide poisoning in male rat. Drug Chem Toxicol 39: 375-379, 2016.

3. Hashemzaei M, Karami SP, Delaramifar A, Sheidary A, Tabrizian K, Rezaee R, Shahsavand S, Arsene AL, Tsatsakis AM and Taghdisi SM: Anticancer effects of co-administration of daunorubicin and resveratrol in MOLT-4, U266 B1 and Raji cell lines. Farmacia 64: 36-42, 2016.

4. Tabeshpour J, Hashemzaei M and Sahebkar A: The regulatory role of curcumin on platelet functions. J Cell Biochem 119: 8713-8722, 2018

5. Tabrizian K, Yaghoobi NS, Iranshahi M, Shahraki J, Rezaee R and Hashemzaei M: Auraptene consolidates memory, reverses scopolamine-disrupted memory in passive avoidance task, and ameliorates retention deficits in mice. Iran J Basic Med Sci 18: 1014-1019, 2015.

6. Buijsse B, Weikert C, Drogan D, Bergmann M and Boeing H: Chocolate consumption in relation to blood pressure and risk of cardiovascular disease in German adults. Eur Heart J 31: 1616-1623, 2010.

7. Di Castelnuovo A, Rotondo S, Iacoviello L, Donati MB and De Gaetano G: Meta-analysis of wine and beer consumption in relation to vascular risk. Circulation 105: 2836-2844, 2002.

8. Mink PJ, Scrafford CG, Barraj LM, Harnack L, Hong CP, Nettleton JA and Jacobs DR Jr: Flavonoid intake and cardiovascular disease mortality: A prospective study in postmenopausal women. Am J Clin Nutr 85: 895-909, 2007.

9. Peters U, Poole C and Arab L: Does tea affect cardiovascular disease? A meta-analysis. Am J Epidemiol 154: 495-503, 2001.

10. Auclair S, Milenkovic D, Besson C, Chauvet S, Gueux E, Morand C, Mazur A and Scalbert A: Catechin reduces atherosclerotic lesion development in apo E-deficient mice: A transcriptomic study. Atherosclerosis 204: e21-e27, 2009.

11. Norata GD, Marchesi P, Passamonti S, Pirillo A, Violi F and Catapano AL: Anti-inflammatory and anti-atherogenic effects of cathechin, caffeic acid and trans-resveratrol in apolipoprotein E deficient mice. Atherosclerosis 191: 265-271, 2007.

12. Rezaee R, Jamialahmadi K, Riahi Zanjani B, Mahmoudi M, Abnous K, Zamani Taghizadeh Rabe S, Tabasi N, Zali M, Rezaee M, Amin B, et al: Crocin effects on human myeloma cells regarding intracellular redox state, DNA fragmentation, and apoptosis or necrosis profile. Jundishapur J Nat Pharm Prod 9: e20131, 2014

13. Rezaee R, Mahmoudi M, Abnous K, Zamani Taghizadeh Rabe S, Tabasi N, Hashemzaei M and Karimi G: Cytotoxic effects of crocin on MOLT-4 human leukemia cells. J Complement Integr Med 10: 105-112, 2013.

14. Yaribeygi H, Mohammadi MT, Rezaee R and Sahebkar A: Crocin improves renal function by declining Nox-4, IL-18, and p53 expression levels in an experimental model of diabetic nephropathy. J Cell Biochem 119: 6080-6093, 2018.

15. Lee IA, Lee JH, Baek NI and Kim DH: Antihyperlipidemic effect of crocin isolated from the fructus of Gardenia jasminoides and its metabolite Crocetin. Biol Pharm Bull 28: 2106-2110, 2005. 
16. Mashmoul M, Azlan A, Mohtarrudin N, Nisak B, Yusof M and Khaza'ai H: Saffron extract and crocin reduced biomarkers associated with obesity in rats fed a high-fat diet. Mal J Nutr 23: 117-127, 2017.

17. Anaeigoudari A, Faramarzi A, Abbasnezhad A and Shafei M: Effect of intrapertonal injection of crocin on cardiovascular parameters in Angiotensin II-induced hypertensive rats. Horizon Med Sci 24: 309-315, 2018

18. Imenshahidi M, Razavi BM, Faal A, Gholampoor A, Mousavi SM and Hosseinzadeh $\mathrm{H}$ : Effects of chronic crocin treatment on desoxycorticosterone acetate (doca)-salt hypertensive rats. Iran J Basic Med Sci 17: 9-13, 2014

19. Imenshahidi M, Hosseinzadeh $H$ and Javadpour Y: Hypotensive effect of aqueous saffron extract (Crocus sativus L.) and its constituents, safranal and crocin, in normotensive and hypertensive rats. Phytother Res 24: 990-994, 2010.

20. He SY, Qian ZY and Tang FT: Effect of crocin on intracellular calcium concentration in cultured bovine aortic smooth muscle cells. Yao Xue Xue Bao 39: 778-781, 2004 (In Chinese)

21. Williams BA, Liu C, Deyoung L, Brock GB and Sims SM: Regulation of intracellular $\mathrm{Ca}^{2+}$ release in corpus cavernosum smooth muscle: Synergism between nitric oxide and cGMP. Am J Physiol Cell Physiol 288: C650-C658, 2005.

22. Iranshahi M, Rezaee R, Parhiz H, Roohbakhsh A and Soltani F: Protective effects of flavonoids against microbes and toxins: The cases of hesperidin and hesperetin. Life Sci 137: 125-132, 2015.

23. Parhiz H, Roohbakhsh A, Soltani F, Rezaee R and Iranshahi M: Antioxidant and anti-inflammatory properties of the citrus flavonoids hesperidin and hesperetin: An updated review of their molecular mechanisms and experimental models. Phytother Res 29: 323-331, 2015.

24. Rezaee R, Sheidary A, Jangjoo S, Ekhtiary S, Bagheri S, Kohkan Z, Dadres M, Oana Docea A, Tsarouhas K, Sarigiannis DA, et al: Cardioprotective effects of hesperidin on carbon monoxide poisoned in rats. Drug Chem Toxicol: Aug 14, 2019 (Epub ahead of print).

25. Roohbakhsh A, Parhiz H, Soltani F, Rezaee R and Iranshahi M: Neuropharmacological properties and pharmacokinetics of the citrus flavonoids hesperidin and hesperetin - a mini-review. Life Sci 113: 1-6, 2014

26. Roohbakhsh A, Parhiz H, Soltani F, Rezaee R and Iranshahi M: Molecular mechanisms behind the biological effects of hesperidin and hesperetin for the prevention of cancer and cardiovascular diseases. Life Sci 124: 64-74, 2015.

27. Haidari F, Heybar H, Jalali MT, Ahmadi Engali K, Helli B and Shirbeigi E: Hesperidin supplementation modulates inflammatory responses following myocardial infarction. J Am Coll Nutr 34: 205-211, 2015

28. Homayouni F, Haidari F, Hedayati M, Zakerkish M and Ahmadi K: Blood pressure lowering and anti-inflammatory effects of hesperidin in type 2 diabetes; a randomized double-blind controlled clinical trial. Phytother Res 32: 1073-1079, 2018.

29. Morand C, Dubray C, Milenkovic D, Lioger D, Martin JF, Scalbert A and Mazur A: Hesperidin contributes to the vascular protective effects of orange juice: A randomized crossover study in healthy volunteers. Am J Clin Nutr 93: 73-80, 2011.
30. Demonty I, Lin Y,Zebregs YE, Vermeer MA, van der Knaap HC, Jäkel $M$ and Trautwein EA: The citrus flavonoids hesperidin and naringin do not affect serum cholesterol in moderately hypercholesterolemic men and women. J Nutr 140: 1615-1620, 2010.

31. Rizza S, Muniyappa R, Iantorno M, Kim JA, Chen $H$, Pullikotil P, Senese N, Tesauro M, Lauro D, Cardillo C, et al: Citrus polyphenol hesperidin stimulates production of nitric oxide in endothelial cells while improving endothelial function and reducing inflammatory markers in patients with metabolic syndrome. J Clin Endocrinol Metab 96: E782-E792, 2011.

32. Ohtsuki K, Abe A, Mitsuzuwi H, Kondo M, Uemura K, Iwasaki Y and Kondo Y: Effects of long-term administration of hesperidin and glucosyl hesperidin to spontaneously hypertensive rats. J Nutr Sci Vitaminol (Tokyo) 48: 420-422, 2002.

33. Yamamoto M, Suzuki A and Hase T: Short-term effects of glucosyl hesperidin and hesperetin on blood pressure and vascular endothelial function in spontaneously hypertensive rats. J Nutr Sci Vitaminol (Tokyo) 54: 95-98, 2008.

34. Asgharpour A, Cazanave SC, Pacana T, Seneshaw M, Vincent R, Banini BA, Kumar DP, Daita K, Min H-K, Mirshahi F, et al: A diet-induced animal model of non-alcoholic fatty liver disease and hepatocellular cancer. J Hepatol 65: 579-588, 2016.

35. Lin HT, Shiou YL, Jhuang WJ and Lee HC: Simultaneous electrocardiography recording and invasive blood pressure measurement in rats. J Vis Exp 143: e59115, 2019.

36. Parasuraman S and Raveendran R: Measurement of invasive blood pressure in rats. J Pharmacol Pharmacother 3: 172-177, 2012.

37. Dobrian AD, Davies MJ, Prewitt RL and Lauterio TJ: Development of hypertension in a rat model of diet-induced obesity. Hypertension 35: 1009-1015, 2000.

38. Ikemura M, Sasaki Y, Giddings JC and Yamamoto J: Preventive effects of hesperidin, glucosyl hesperidin and naringin on hypertension and cerebral thrombosis in stroke-prone spontaneously hypertensive rats. Phytother Res 26: 1272-1277, 2012.

39. Wunpathe C, Potue P, Maneesai P, Bunbupha S, Prachaney P, Kukongviriyapan U, Kukongviriyapan V and Pakdeechote P: Hesperidin suppresses renin-angiotensin system mediated NOX2 over-expression and sympathoexcitation in $2 \mathrm{~K}-1 \mathrm{C}$ hypertensive rats. Am J Chin Med 46: 751-767, 2018.

40. Shafei MN, Faramarzi A, Khajavi Rad A and Anaeigoudari A: Crocin prevents acute angiotensin II-induced hypertension in anesthetized rats. Avicenna J Phytomed 7: 345-352, 2017.

41. Imenshahidi M, Razavi BM, Faal A, Gholampoor A, Mousavi SM and Hosseinzadeh $\mathrm{H}$ : The effect of chronic administration of saffron (Crocus sativus) Stigma aqueous extract on systolic blood pressure in rats. Jundishapur J Nat Pharm Prod 8: 175-179, 2013.

42. Llorens S, Mancini A, Serrano-Díaz J, D'Alessandro AM, Nava E, Alonso GL and Carmona M: Effects of crocetin esters and crocetin from Crocus sativus L. on aortic contractility in rat genetic hypertension. Molecules 20: 17570-17584, 2015.

43. Mancini A, Serrano-Díaz J, Nava E, D'Alessandro AM, Alonso GL, Carmona M and Llorens S: Crocetin, a carotenoid derived from saffron (Crocus sativus L.), improves acetylcholine-induced vascular relaxation in hypertension. J Vasc Res 51: 393-404, 2014. 